\title{
Multiple zone multifocal phase designs
}

\author{
Pablo de Gracia, Carlos Dorronsoro, and Susana Marcos* \\ Instituto de Óptica, Consejo Superior de Investigaciones Científicas, Madrid 28006, Spain \\ ${ }^{*}$ Corresponding author: susana@io.cfmac.csic.es
}

Received June 21, 2013; revised July 17, 2013; accepted August 2, 2013;

posted August 7, 2013 (Doc. ID 192736); published September 5, 2013

\begin{abstract}
New multifocal phase designs aiming at expanding depth of focus in the presbyopic eye are presented. The designs consist of multiple radial or angular zones of different powers or of combined low- and high-order aberrations. Multifocal performance was evaluated in terms of the dioptric range for which the optical quality is above an appropriate threshold, as well as in terms of the area under the through-focus optical quality curves. For varying optical power designs optimal through-focus performance was found for a maximum of three to four zones. Furthermore adding more zones decreased the optical performance of the solution. Angular zone designs provided better multifocal performance (1.95 times on average) than radial zone designs with identical number of zones and the same power range. The optimal design (angular design with three zones) surpassed by $33 \%$ the multifocal performance of a bifocal angular zone design and by $32 \%$ a standard multifocal phase plate with induced spherical aberration only. By using combinations of low- and high-order aberrations the through-focus range can be extended further by another $0.5 \mathrm{D}$ beyond that of the best design of varying optical power. These designs can be implemented in adaptive optics systems for testing their visual performance in subjects and converted into multifocal contact lenses, intraocular lenses, or presbyopic corneal laser ablation profiles. (c) 2013 Optical Society of America

OCIS codes: (330.1070) Vision - acuity; (330.1800) Vision - contrast sensitivity; (330.4300) Vision system - noninvasive assessment; (330.4460) Ophthalmic optics and devices; (330.7322) Visual optics, accommodation; (330.7323) Visual optics, aging changes.

http://dx.doi.org/10.1364/OL.38.003526
\end{abstract}

The young human eye has the capability of dynamically focusing far and near objects. However with age, the crystalline lens loses this ability to accommodate (presbyopia). There are several solutions available to treat presbyopia and restore some near-vision functionality. These include optical aids (in the form of contact lenses, intraocular lenses, or corneal laser treatments) that aim at producing multiple foci or at expanding depth of focus (DOF) [1]. Simultaneous vision lenses include bifocal or trifocal diffractive and refractive designs, or aspheric designs that generally attempt to modulate the spherical aberration of the eye. Refractive designs show different optical zones of different refractive power, normally concentrically in two zones (for example with the central portion providing near vision and the peripheral one providing far vision), multiple zones, or asymmetric zones (with, for example, the upper zone providing distance vision and the lower zone providing near vision) $[2,3]$. Several studies have proposed expanding DOF by increasing the optical aberrations or by introducing specific combinations of aberrations (i.e., fourth- and sixth-order spherical aberration Zernike terms) [ㄴ, 5$]$. Some other DOF expansion strategies inspired by beam shaping or imaging (i.e., axicons) have encountered limitations for applications in the eye [6]. Recently, multifocal intraocular lens designs with aspheric optics have been proposed based on a multiconfiguration approach, where the optical quality of the eye plus lens is optimized for multiple foci [7]. Many studies propose the construction of a phase pattern (generally defined by a set of aberrations) that optimize a certain visual quality metric [for example the visual Strehl computed from the optical transfer function (OTF)] over a certain dioptric range [8]. Besides optical predictions, it is possible to simulate visual performance with these designs with the use of adaptive optics (AO) simulators. Deformable mirrors are capable of reproducing smooth phase patterns (such as those obtained by combination of aberrations). Spatial light modulators can also repro- duce steep phase changes such as those produced in certain refractive multifocal designs [9]. Despite the availability of technology to produce phase patterns that combine both segmented regions of different powers or aberration profiles, the multifocal optical (or visual) performance produced by those patterns has never been explored systematically. In this study, we numerically simulated the predicted through-focus optical quality of multifocal phase designs consisting of segmented pupils ( $N$ zones up to 50) of progressive power in different radial or angular pattern configurations, where the dioptric power in each zone is defined by

$$
D_{i}=D_{f}+\left(\frac{D_{n}-D_{f}}{N}\right)(i-1),
$$

where $i$ is the zone number, $N$ the total number of zones, $D_{f}$ is the optical correction for far, and $D_{n}$ is the optical correction for near.

In general, the phase pattern $\left(W_{T}\right)$ is defined by the wavefront in each zone, which can be expressed mathematically by

$$
W_{T}=\sum_{i=1}^{N} w_{i}\left(D_{i}\right) * \sigma_{j l},
$$

where $N=n_{1} * n_{2}, l$ labels the radial zones $\left(1=0\right.$ to $\left.n_{1}\right)$, $j$ labels the angular zones $\left(j=0\right.$ to $\left.n_{2}\right), w_{i}$ represents the wave aberration in each zone, and $\sigma_{j l}$ represents a mask that equals to 1 in the corresponding zone and 0 elsewhere. For radial patterns, the corresponding masks $\sigma_{j l}$ are defined by

$$
\sigma_{j l}(\rho, \theta)=1 \quad \forall \rho \in\left[\frac{\rho_{m} l}{n_{1}}, \frac{\rho_{m}(l+1)}{n_{1}}\right], 0 \text { otherwise, }
$$

and for angular patterns by 


$$
\sigma_{j l}(\rho, \theta)=1 \quad \forall \theta \in\left[\frac{2 \pi j}{n_{2}}, \frac{2 \pi(j+1)}{n_{2}}\right]+\Phi, \quad 0 \text { otherwise }
$$

where $\Phi$ stands for an angular shift, common to all zones.

Figure 1 illustrates the distribution of segmented zones in several radial and angular designs. For clarification, the separation between zones has been highlighted (except for the 50-zone designs). We denoted the patterns by $N_{\left(n_{1}, n_{2}, \Phi\right)}$, where $n_{1}$ indicates the number of radial zones and $n_{2}$ the number of angular zones. Wave aberrations $w_{i j}$ in the phase patterns of Fig. 1 are defined by defocus term only [Eq. (1)]. Other phase patterns considered in the study included $w_{i j}$ defined by combinations of high-order aberrations (see Figs. $\underline{4}$ and 5 ).

Fourier optics were used to compute the OTF from the pupil function. The visual Strehl (obtained as the volume between the OTF and a general neural transfer function to emphasize the spatial frequency range most relevant to visual function) was used as an optical quality metric $[8,10]$. The threshold for acceptable vision was set to 0.12 visual Strehl, as reported in prior literature $[\underline{4}, \underline{11}]$. Through-focus visual Strehl curves were computed to evaluate the through-focus performance of the designed phase patterns. DOF was defined as the dioptric range for which visual Strehl was above threshold. Also, the area under the visual Strehl (in a dioptric range of $6 \mathrm{D}$ ) was used as an optical quality metric.

Current clinically available multifocal refractive intra ocular lens (IOL) designs use various concentric segmented zones $N_{(N, 0,0)}$ according to our notation, that is, AT Lisa bifocal lens by Carl Zeiss or the ReZoom bifocal lens by Abbot $5_{(5,1,0)}$. To our knowledge only one IOL design uses roughly a two-angular-zone design [MPlus lens, by Oculentis, which in a first approximation could be described by $\left.2_{(1,2,0)}\right][\underline{12}]$. Although in some multizonal lens designs aspheric transition zones are included to facilitate smooth variations across zones with different power, multifocality is mostly produced by differences in power in the different zones. However, the optimal number of zones in radial and angular zone designs, and potential differences in optical performance of radial versus angular designs (with equal number of zones and area of the corresponding zones of similar power), have never been tested.

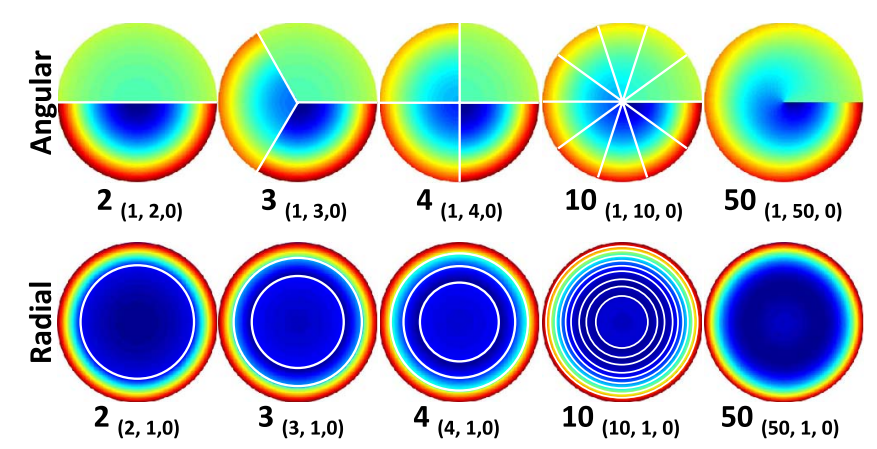

Fig. 1. $W_{T}$ for different numbers of angular (top row) and radial (lower row) zones. Maps are represented considering only defocus in angular and radial designs. Values of $\left[n_{1}, n_{2}, \Phi\right]$ are shown for each pattern.
We designed multifocal phase patterns with $N$ zones both with optical power varying in radial $\left(N_{(N, 0,0)}\right)$ and angular $\left(N_{(0, N, 0)}\right)$ configurations, with $N$ ranging from 1 to 50 . The defocus term $\left(C_{0}^{2}\right.$ in a Zernike expansion notation) was varied linearly and sequentially across zones between -0.2 and $-1.7 \mu \mathrm{m}$ in a $4 \mathrm{~mm}$ pupil, equivalent to a dioptric power change from $+0.35 \mathrm{D}$ for far distance correction to $+3 \mathrm{D}$ for near (i.e., near addition). The area of each zone is of equal value in all cases $\left(\pi^{*} \rho^{2} / N \mathrm{~mm}^{2}\right)$. Figure $\underline{2}$ shows the visual Strehl-based optical performance metrics (DOF versus area under the visual Strehl through-focus curve) for radial and angular zone designs of increasing number of zones (up to $N=50$ ), always with power varying by $2.65 \mathrm{D}$ from the far to near zones. The threshold level for determining the DOF is 0.12 . Interestingly, in both cases, increasing the number of zones does not lead to an increase in performance. The best multifocal optical performance (large DOF while preserving a high area under the curve) corresponds to three to four zones, in both radial and angular zone designs. Figure 3 shows the visual Strehl through-focus curves for selected power-varying designs with radial zones and angular zones, respectively. In radial zone designs, increasing the number of zones will eventually lead to a spherical aberration multifocal profile, as shown in Fig. 3 for a $1_{(1,0,0)}$ pupil pattern, where wave aberration $W_{T}$ is defined by a fourth-order spherical aberration $\left(\mathrm{C}_{0}^{4}=0.4 \mu \mathrm{m}\right)$. Remarkably, this solution (although frequently proposed $[\underline{4}, \underline{13}])$ provides poorer optical performance than a bifocal solution and is largely exceeded by a trifocal solution (trifocal diffractive IOL designs have been recently released commercially $[\underline{14}, \underline{15}])$. In angular zone designs, increasing the number of zones will eventually lead to a spiral phase plate (or optical vortex), which has been proposed in other areas as a focal beam expander [16], although they appear suboptimal in the current application. Currently clinically available approaches using two angular zones (upper for far and lower for near) could be improved by increasing the number of zones to 3 .

Interestingly, the optical performance of radial and angular zone designs differs, even for similar number of zones $(N)$, similar power values in each zone, and

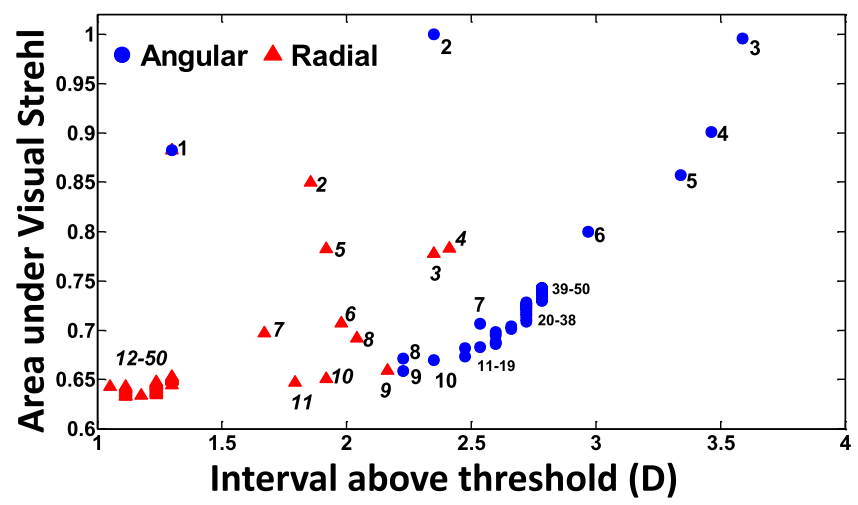

Fig. 2. DOF versus area under the visual Strehl through-focus curves, for phase patterns with $N$ angular (blue circles) and radial (red triangles) zones ( $N$ ranges from 1 to 50 ). The labels next to each spot stand for the number of zones of the corresponding design. Area under the visual Strehl is normalized by the area of the bifocal angular design. Data are for $4 \mathrm{~mm}$ pupil diameter. 


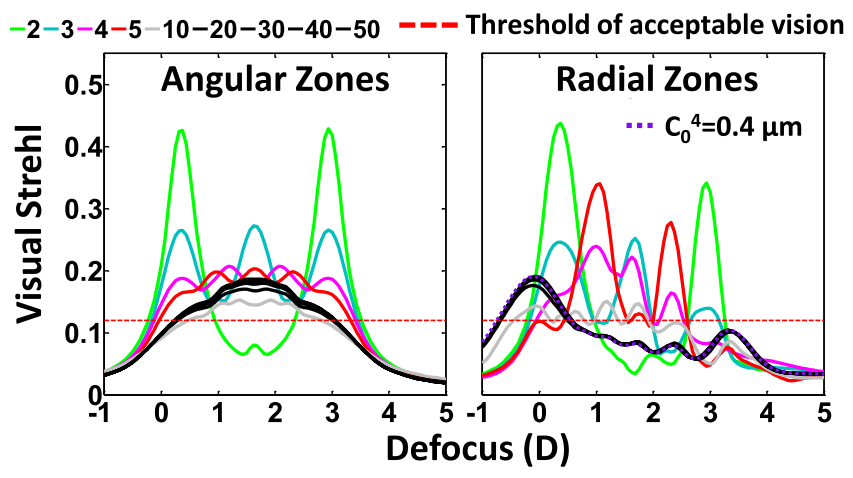

Fig. 3. Visual Strehl value as a function of defocus for phase patterns generated with $2,3,4,5,10,20,30,40$, and 50 zones. Left panel: phase patterns with angular zones. Right panel: phase patterns with radial zones and $C_{0}^{4}=0.4 \mu \mathrm{m}$. Data are for $4 \mathrm{~mm}$ pupil diameter.

similar zone areas, indicating that the actual zonal distribution matters. Results revealed that for the same number of zones angular designs are more efficient than radial designs ( $12 \%$ on average across the 50 designs). The number of zones in the angular design directly translates into increased number of peaks in the through-focus curve (see Fig. 3). However, a less predictable behavior occurs with radial designs, which ultimately (for large $N$ ) become similar to a spherical aberration phase pattern. An additional advantage of angular designs includes more independence of their performance with the pupil size.

These results suggest that phase patterns with angularly segmented zones (two to four) are optimal in expanding DOF while preserving acceptable visual performance. Our analyses up to this point have only considered differences in power across different zones. Prior studies have reported that interactions between high-order aberrations occur, which may lead to increased optical quality $[5,11]$. It is likely that combinations of aberrations other than defocus in each zone also have a positive impact on the through-focus optical quality of multifocal phase patterns.

We have generated six angular zone multifocal phase patterns (Fig. 4), with $2_{(1,2,0)}$ (designs A through C) and $4_{(1,4, \pi / 4)}$ zone patterns (designs D through F) and introduced different combinations of aberrations in each zone. The second column in Fig. 4 shows the selected Zernike coefficients for each zone of designs A through $\mathrm{F}$. The through-focus performance of these designs is shown in Fig. 5.

The introduction of spherical aberration to expand DOF has been studied before $[4,13]$. A control condition of $0.22 \mu \mathrm{m}$ of spherical aberration and $0.8 \mu \mathrm{m}$ of defocus (this combination of spherical aberration and defocus allowed peak performance for emmetropic patients [4]) has been included for a direct comparison with the current state of the art. Pattern A is designed by a combination of positive spherical aberration $(0.22 \mu \mathrm{m})$ and positive defocus $(0.8 \mu \mathrm{m})$ in zone 1 and the same amounts of spherical aberration and defocus but with a reversed sign of the spherical aberration term in zone 2 . This configuration produced a $10 \%$ increase in DOF in comparison to the same amount of only spherical aberration across the entire pupil (see Fig. 5A). Favorable interactions between
Zernike Coef.

\section{A

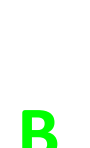

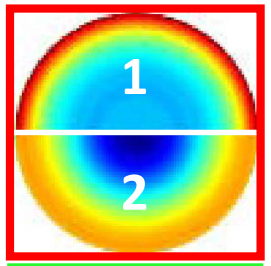
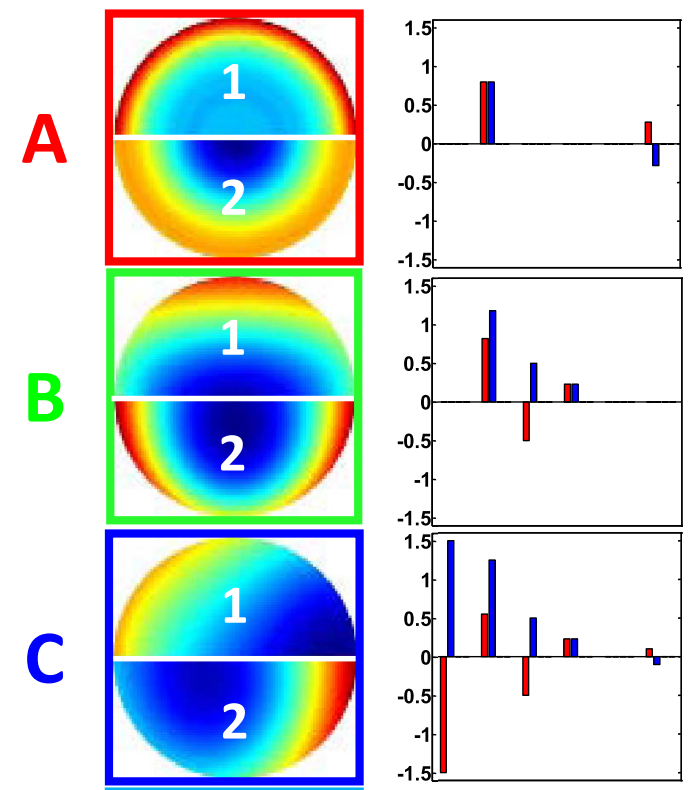

$-1.5$
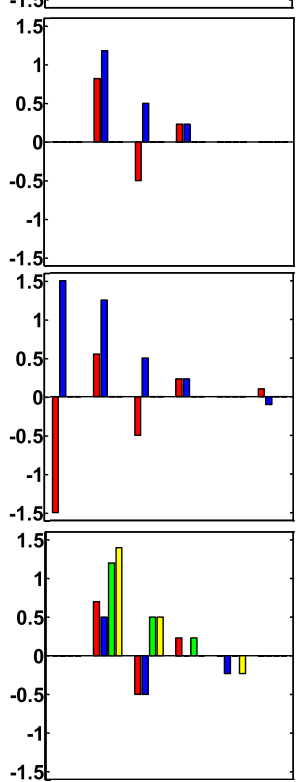

$-1.5$
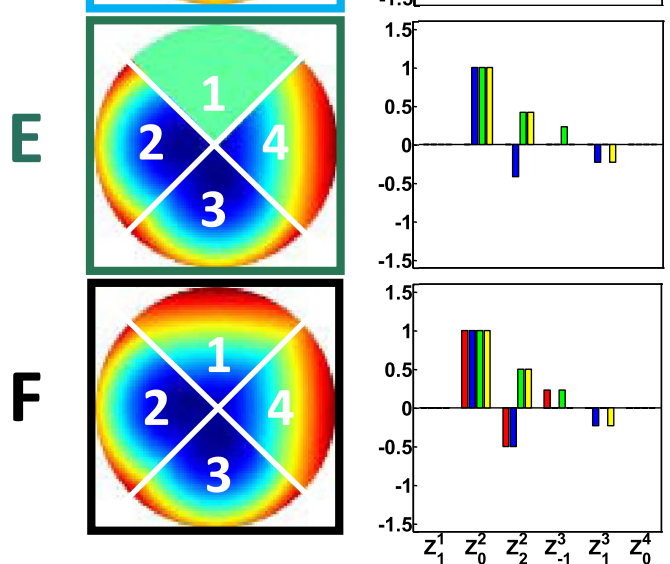

Zone $1 \square$ Zone $2 \square$ Zone $3 \square$ Zone 4

Fig. 4. Left column: Zernike coefficients of the wave aberration in each zone for each of the six multifocal phase patterns. Right column: multifocal phase patterns generated by the combination of high- and low-order aberrations. Data are for $4 \mathrm{~mm}$ pupil diameters.

astigmatism and coma have been found under certain conditions in previous works [5,17]. Patterns B, C, D, E, and F involve the reported optimal combinations of defocus, astigmatism, and coma which increased optical performance in monofocal vision with respect to astigmatism alone [5]. A particular four-angular-zone design with combined astigmatism and coma in each zone (Pattern D) produced the largest DOF ( $>4 \mathrm{D}$ ) while keeping the area under the through-focus visual Strehl close to 1 (see Fig. 5B).

In summary, our study demonstrates that multizonal angular phase patterns with three to four zones are optimal. The theoretical performance of multifocal designs 

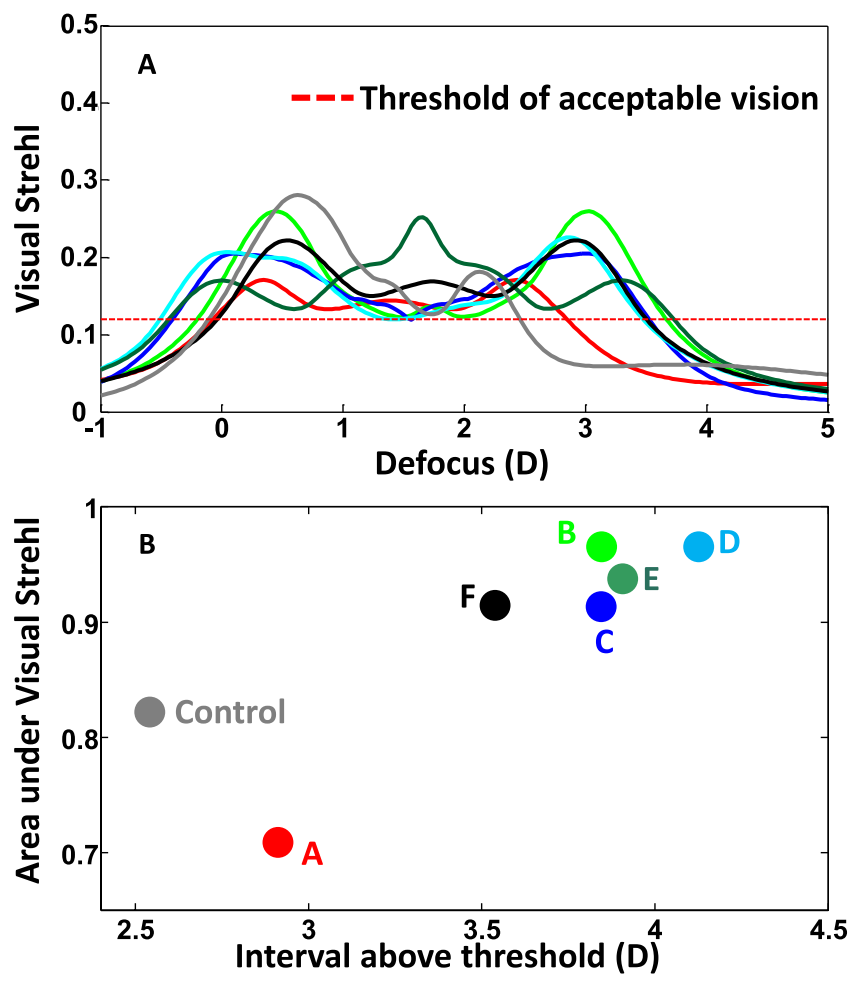

Fig. 5. (A) Through-focus visual Strehl for the six different multifocal designs described in Fig. 4 (corresponding colors) with multiple zones and different combinations of aberrations in each zone. The gray line represents values for a 1-zone multifocal pattern with spherical aberration $(0.22 \mu \mathrm{m})$. (B) DOF versus area under the visual Strehl through-focus curves for the six designs described in Fig. 4. Area is normalized by that of the bifocal angular design. Data are for $4 \mathrm{~mm}$ pupil diameters.

with three angular zones of different power expanded DOF $40 \%$ more than current angular bifocal designs, $40 \%$ more than trifocal radial designs, and $32 \%$ more than a typical spherical aberration phase pattern [4]. For the three-zone angular design through-focus optical quality also varied with respect to the mentioned conventional designs by $-1 \%, 14 \%$, and $23 \%$, respectively. Our study demonstrates that multizonal angular phase patterns with three to four zones are optimal. To our knowledge, there is no multifocal lens available with this configuration, but current IOL and contact lens designs could be improved by combining standard IOL and contact lens design approaches with the results of this study.

In addition (see Fig. 5B), the dioptric range above threshold can be extended up to $0.5 \mathrm{D}$ by introducing combinations of other aberrations other than defocus with respect to the power-varying trifocal angular design (Fig. 2).

Vision through the presented multifocal phase patterns can be experimentally simulated in AO systems provided with spatial light modulators. Although the visual Strehl used in the theoretical computations has been shown to correlate best with visual performance [8]], the current analysis has been performed purely on optical grounds.

Experimental AO simulations will incorporate neural factors, interactions of the multifocal phase pattern with the ocular aberrations of the subject, and potentially the prior visual experience (spatial neural adaptation) of subjects [18]. The designed phase patterns can be transferred to surface profiles in contact lenses and intraocular lenses, or implemented in refractive surgery ablation profiles, with the required considerations specific to each correction alternative The implementation of these phase patterns onto contact lenses or presbyopic laser corrections will require transfer of the wave aberration at the pupil plane to the corneal plane and defining the corresponding tissue ablation profile (in corneal refractive surgery) [19] and lens geometry (in contact lenses) [20]. Other relevant aspects include laser ablation efficiency (in corneal refractive surgery) [21], tear lens effects (in contact lenses) [22], and IOL lens geometry and platform (in IOLs) [23].

The research leading to these results has received funding from the European Research Council under the European Union's Seventh Framework Programme (FP7/2007-2013)/ERC Grant Agreement No. 294099, Spanish Government Grant FIS2011-25637 to Susana Marcos and from two Ezell Fellowships conceded by the American Academy of Optometry to Pablo de Gracia.

\section{References}

1. A. Glasser, M. A. Croft, and P. L. Kaufman, Int. Ophthalmol. Clin. 41, 1 (2001).

2. A. Lichtinger and D. S. Rootman, Curr. Opin. Ophthalmol. 23, 40 (2012).

3. J. A. Martin and A. Roorda, Optom. Vis. Sci. 80, 812 (2003).

4. F. Yi, D. Robert Iskander, and M. Collins, Vis. Res. 51, 1648 (2011).

5. P. de Gracia, C. Dorronsoro, E. Gambra, G. Marin, M. Hernández, and S. Marcos, Vis. Res. 50, 2008 (2010).

6. A. Zlotnik, I. Raveh, S. Ben Yaish, O. Yehezkel, M. Belkin, and Z. Zalevsky, Proc. SPIE 7550, 75500A (2010).

7. D. Fernández, S. Barbero, C. Dorronsoro, and S. Marcos, "Multifocal intraocular lens providing improved," Spanish patent P201232043 (December 27, 2012).

8. J. D. Marsack, L. N. Thibos, and R. A. Applegate, J. Vis. 4(4), 8 (2004).

9. S. Manzanera, P. M. Prieto, D. B. Ayala, J. M. Lindacher, and P. Artal, Opt. Express 15, 16177 (2007).

10. D. R. Iskander, Optom. Vis. Sci. 83, 57 (2006).

11. X. Cheng, A. Bradley, and L. N. Thibos, J. Vis. 4(4), 310 (2004).

12. B. F. M. W. Angerlo and B. J. W. Arnhem, "Ophthalmic lens with optical sectors,” U.S. patent application 20120029631 A1 (May 9, 2012).

13. Y. Benard, N. Lopez-Gil, and R. Legras, Vis. Res. 51, 2471 (2011).

14. W. Fiala and M. Gerlach, "Multifocal lens," U.S. patent application 20130050640 (October 28, 2012).

15. D. Gatinel, C. Pagnoulle, Y. Houbrechts, and L. Gobin, J. Cataract. Refract. Surg. 37, 2060 (2011).

16. W. M. Lee, X. C. Yuan, and W. C. Cheong, Opt. Lett. 29, 1796 (2004).

17. P. de Gracia, C. Dorronsoro, G. Marin, M. Hernández, and S. Marcos, J. Vis. 11(2), 5 (2011).

18. L. Sawides, P. de Gracia, C. Dorronsoro, M. A. Webster, and S. Marcos, PLoS ONE 6, e27031 (2011).

19. S. Klein, J. Opt. Soc. Am. A 15, 2580 (1998).

20. S. A. Klein and B. A. Barsky, Optom. Vis. Sci. 72, 816 (1995).

21. C. Dorronsoro, D. Cano, J. Merayo, and S. Marcos, Opt. Express 14, 6142 (2006).

22. C. Dorronsoro, S. Barbero, L. Llorente, and S. Marcos, Optom. Vis. Sci. 80, 115 (2003).

23. M. Bass, ed., Handbook of Optics, 3rd ed. (Optical Society of America, 1995), Vol. 3, Chap. 21. 